\title{
Singular peculiarities of a plane wave diffracted on half-plane
}

\author{
S. Anokhov'), A. Khizhnyak ${ }^{2)}$, R.Lymarenko'), M. Soskin ${ }^{3)}$ \\ ${ }^{1}$ International Center "Institute of Applied Optics», National Academy of Sciences of Ukraine, 04053, Kyiv , Ukraine \\ phone: 380(44)2122158,fax380(44)2124812,e-mail:anokhov@i.kiev.ua; \\ ${ }^{2}$ MetroLaser, Inc. Skypark Circle, Suite 100, Irvine, CA, 92614-6428, USA, e-mail: khizh@metrolaserinc.com, \\ 3) "Institute of Physics" National Academy of Sciences of Ukraine, Prospect Nauki, 46, 02650, Kiev, Ukraine, \\ e-mail:marats@vortex.kiev.ua.
}

Abstract. We analyzed singular properties of edge dislocation waves («EDW») - the main information component of the field formed at plane wave diffraction on half-plane. It is shown that analytical structure of this wave is completely identical to Cornu's spiral, while physical simulation thereof requires joining the plane wave with an edge dislocation. Dislocations of this type are rather sensitive to action of any amplitude phase distortion on them that considering the impact of actual noise essentially hampers their experimental isolation in the pure form. At the same time spatial position of such dislocation may be effectively controlled by changing the amplitude and phase of one of the wave components. We considered peculiarities of structural evolution of the field at more complicated form of diffraction aperture.

Keywords: diffraction, singular optics, half-plane, edge dislocation

Paper received 27.04.01; revised manuscript received 04.07.01; accepted for publication 13.07.01.

\section{Introduction}

As we shown recently $[1,2]$, rigorous strict solution for plane wave diffraction on half-plane obtained by Sommerfeld more than 100 years ago allows more convenient wave interpretation thereof, which is free from inconsistencies inherent to the model traditionally used in this case [3]. In particular, appearance of the field in a shadow area at new interpretation is related with existence of actual wave, qualitatively different by its structure from the Young boundary edge wave and easily isolated in experiment. A peculiarity inherent to this wave is availability of wavefront at its edge dislocation spatially coinciding with the edge of the geometrical shadow. Extraordinary properties of such wave, in essence, are to all inherent peculiarities of the diffraction process.

The purpose of the present work is to analyse singular properties of the said above wave beyond the framework of the preceding publications essentially important for understanding the diffraction field structure.

\section{New wave model of diffraction field}

The geometry of the task solved by Sommerfeld is presented in Fig. 1. We have shown that according to the rigorous strict solution, the field formed behind the halfplane, may be represented by a set of two independent waves whose distribution direction coincides with that of the initial wave. In particular, at diffraction of $Z$-polarized plane wave, this solution is easily converted to [1,2]:

$$
E_{z}=\left(E_{z}^{(p l)}+E_{z}^{(i n h)}\right) \times \exp \left[-i k r \cos \left(\theta-\alpha_{o}\right)\right],
$$

where the first component is a homogeneous plane wave

$$
E_{z}^{(p l)}=\left(A_{o} / 2\right)
$$

identical to the incident wave but having twice as less an amplitude, and the second is a nonuniform wave

$$
E_{z}^{(i n h)}=\frac{A_{o}(1-i)}{\sqrt{2 \pi}} \times \int_{0}^{U} \exp \left(i \mu^{2}\right) d \mu,
$$




\section{S. Anokhov et al.: Singular peculiarities of a planey wave diffracted ...}

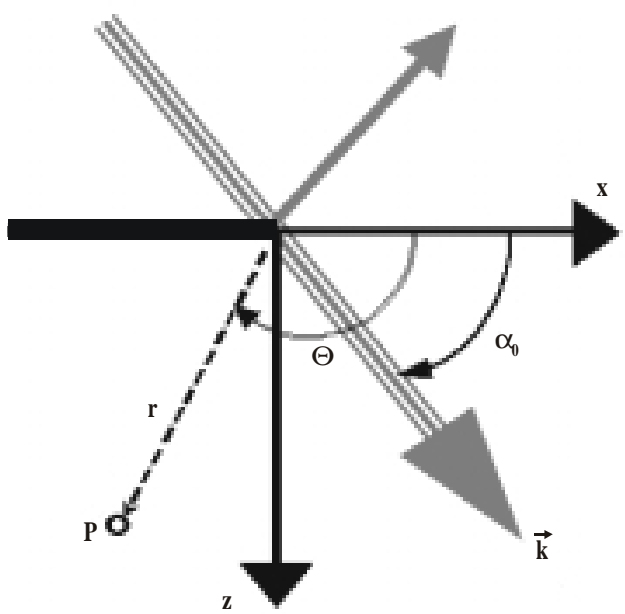

Fig. 1. The geometry of the half-plane screen diffraction: the screen is placed at the plane $y=0, x \geq 0, r$ is the distance between the screen edge and observation point $P ; k$ is the wave vector of the incident plane wave. The angle $\alpha_{0}\left(0 \leq \alpha_{0} \leq \pi\right)$ is measured from the $X$ axis to the incident wave vector direction, the angle $\theta$ is measured from the $X$ axis to the radius-vector direction. $Z$-axis coincides with the screen edge. Mirror-reflected wave direction is also shown.

containing, in essence, all information on the diffraction process [1,2]. Terms used above include: $r=\sqrt{x^{2}+y^{2}}$ is the distance from the edge of the half-plane to the point of observation, $k-$ is the wave number, $U=-\sqrt{2 k r} \cos \frac{\theta-\alpha_{o}}{2}$ is the dimensionless wave parameter.

Fig. 2 contains distribution of amplitude and phase of the nonuniform components in Cartesian coordinates at normal incidence falling of initial wave on half-plane $\left(\alpha_{o}=\pi / 2\right)$, when the wave parameter is $U=\left\{k\left[\left(x^{2}+\right.\right.\right.$ $\left.\left.\left.+y^{2}\right)^{1 / 2}-y\right]\right\}^{1 / 2}$, and, in most important for practice paraxial area, is reduced to $U \approx x \sqrt{\pi / \lambda y}$.

From expression (3), it follows that in the plane $x=0$, coinciding with the edge of the geometrical shadow, the amplitude of nonuniform components zeroes (Fig. 2a), and phase is changed in leaps by $p$ while keeping its derivative continuous (Fig. 2b). This means that on the edge of the geometrical shadow, the nonuniform component has edge dislocation of wavefront, thereupon such wave was named EDW (edge dislocation wave) [1,2]. Fig. 2c, $\mathrm{d}$ show equivalent smooth representation of these dependencies, sometimes more convenient, than the first one, where the phase leap of the field by $\pi$ is transformed into the sign change of the amplitude wave.

Destructive interference of the waves $E_{z}^{(p l)}$ and $E_{z}^{(E D W)}$ in the area of geometrical shadow leads to their fast cross suppression even at small distance from the edge of the shadow. It is the aggregate of the said waves which makes the actual field observed in the shadow area and taken by many for the edge Young wave [3]. The structure of this field with monotonically drop-down from the edge of the shadow amplitude remains constant at any removal of the diffracted wave from the half-plane, undergoing only scale transformation at the same time.

The advantage of the diffraction field description used here consists of essential simplification of the physical model of the phenomena, achieved by substitution of two discontinuous and, therefore, unable to self-maintained existence of the Young waves (geometrical and boundary edge) by two actual waves, which are eligible to simple experimental isolation and consideration [1,2]. Moreover, understanding EDW have appeared to be rather fruitful basis for quantitative analysis of more complicated diffraction phenomena, in particular, diffraction process of arbitrary beam on half-plane [4] and arbitrarily tilted inclined plane wave on slot slit [5 ].

\section{Amplitude-phase structure of EDW}

Let us consider the peculiarities of EDW distinguishing it from the waves normally occurring in optics and having monotonic amplitude-phase distribution in their spa-
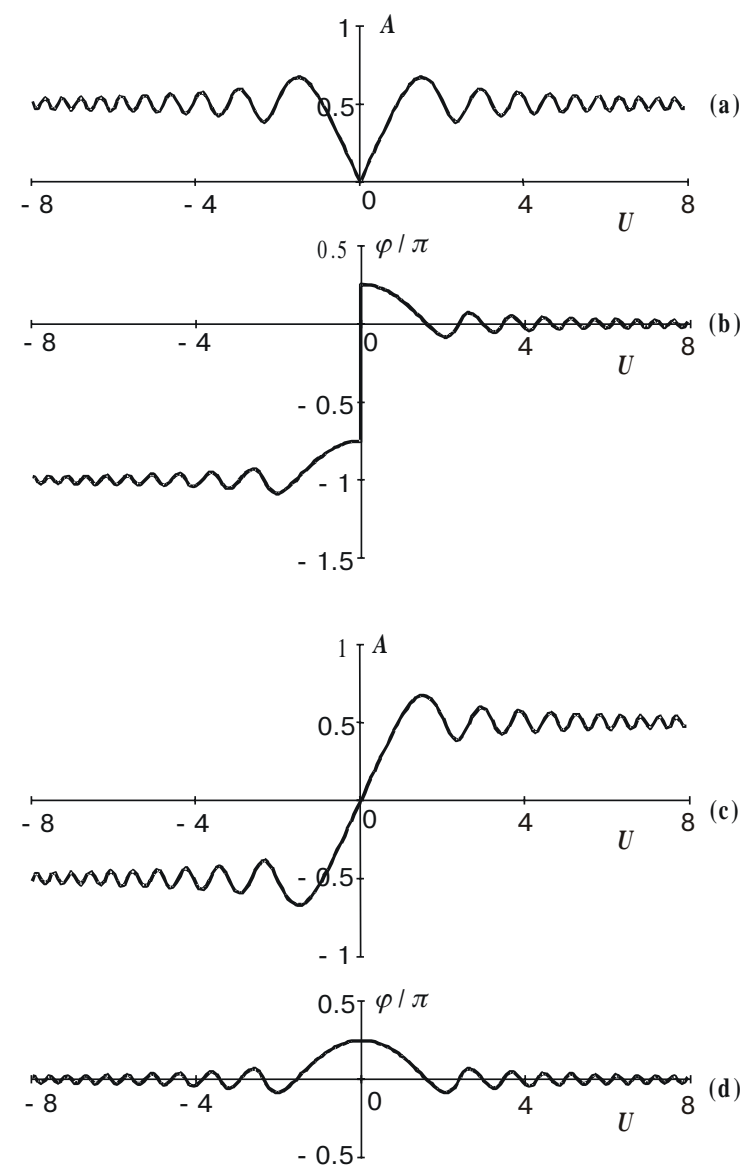

Fig. 2. Amplitude (a) and phase (b) distribution near the geometrical shadow. Equivalent smooth representation of amplitude-phase structure of EDW (c,d). 


\section{S. Anokhov et al.: Singular peculiarities of a planey wave diffracted ...}

tial area. First and foremost, notable is the essentially nonuniform character thereof. The amplitude of EDW, at removal from the edge of the geometrical shadow, undergoes damped oscillations near the constant level, equal to half amplitude of the initial plane wave (Fig. 2). EDW wavefronts in their central part, i.e. in the phase leap area are parabolic, corresponding to the divergent energy stream, and, with passage to periphery, with oscillations, similar to the amplitude ones, transforms into plane. At distribution of EDW in free space, it, owing to its self-similar character, maintains the said structure, experiencing only monotonic scale magnification.

At normal incidence falling of initial wave on halfplane, the position of the EDW amplitude maxima corresponding to the maximum intensity of the aggregate field $E_{z}$, changes in paraxial area as $x_{i} \sim y^{1 / 2}$ (given $U=$ const). Owing to this, their spatial position is described by a set of square-law parabolas, originating close to the screen edge of the screen and oriented lengthways the edge of the geometrical shadow [2]. At arbitrary inclination tilt of the incident wave, the said set transforms into a number of inclined parabolic dependencies

$\left[2 k\left(x^{2}+y^{2}\right)^{1 / 2}\right]^{1 / 2} \cos \left[\left(\pi+\operatorname{arctg}\{\mathrm{y} / \mathrm{x}\}-a_{o}\right) / 2\right]=$ const.

Self-similar character of EDW, naturally, persists following such waves passing through optical filter, eliminating paraxial components of angular spectrum thereof (Curve 2 in Fig. 3). Such «truncated» EDW is normally taken for nonexistent in the nature boundary edge Young wave (Curve 3 in Fig. 3) at attempts of experimental isolation thereof by optical filtration of the diffraction field [3].

The determining role played by EDW in the diffraction process, is much explained by close connection thereof with Cornu's spiral, long used in optics and acoustics for quantitative description of plane waves diffraction on rectilinear edges [3]. Indeed, given the variable substitution in EDW amplitude equation under integral
(3), the following is easily obtained:

$$
E_{z}^{(E D W)}=A_{o} \times\left\{\frac{(1-i)}{2} \times[C(V)+i S(V)]\right\},
$$

where $C(V)=\int_{0}^{V} \cos \left(\frac{\pi t^{2}}{2}\right) d t, S(V)=\int_{0}^{V} \sin \left(\frac{\pi t^{2}}{2}\right) d t$ is the Fresnel integral with the wave parameter $V=U \times \sqrt{\frac{2}{\pi}}$. At normal incidence of initial wave on the screen, we obtain: $V=2 \sqrt{\left(\sqrt{x^{2}+y^{2}}-y\right) / \lambda}$, and, in paraxial area, $V \approx x \sqrt{\frac{2}{\lambda y}}$. According to expression in curly brackets (5), the amplitude distribution of EDW field is described by Cornu's spiral in complex representation [3]. This circumstance allow to specify, essentially for the first time, physical sense of spiral of Cornu's spiral, whose actual embodiment, obviously now, is EDW - the main information component of the diffraction field. Visual acknowledgement of this is given in Fig. 4, which shows the amplitude distribution of EDW, i.e. function (5) in space $\{\mathrm{V}$, $\left.\operatorname{Re}\left[E_{z}^{(E D W)}\right], \operatorname{Im}\left[E_{z}^{(E D W)}\right]\right\}$, describing Cornu's spiral «stretched» in this space. Passing to singular peculiarities of EDW, a special interest is gained by projections of this spiral onto the planes $\operatorname{Re}\left[E_{z}^{(E D W)}\right]=$ const, $\operatorname{Im}\left[E_{z}^{(E D W)}\right]=$ const (Fig. 5).

\section{Singular properties of EDW}

\subsection{Topological structure of edge dislocation}

General sign of edge dislocation availability at wavefront, as it is known, is simultaneous zeroing of both real and imaginary components of the wave's amplitude [6]. In case of EDW, this condition is

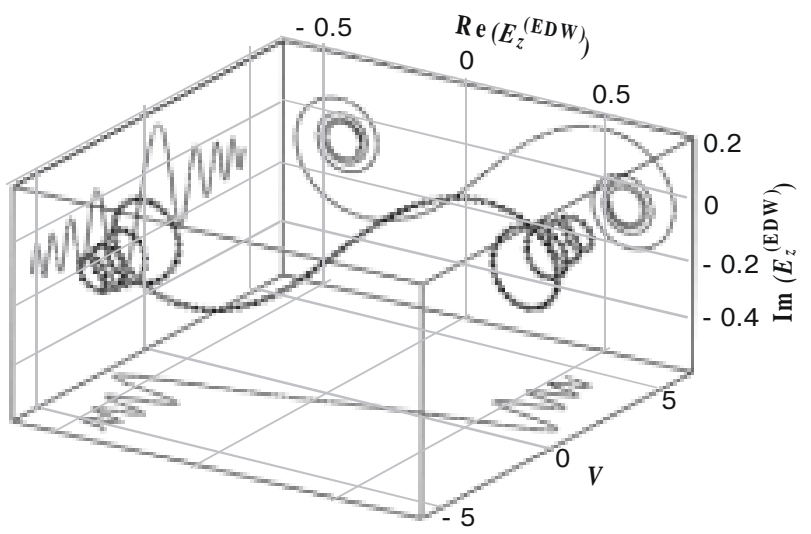

Fig. 4. EDW in coordinates $\left.\left\{\mathrm{V}, \operatorname{Re} E_{z}^{(E D W)}\right], \operatorname{Im}\left[E_{z}^{(E D W)}\right]\right\}$.
Fig. 3. Amplitude distribution of EDW (1), «truncated» EDW (2) and the Young boundary (3) waves 


\section{S. Anokhov et al.: Singular peculiarities of a planey wave diffracted ...}
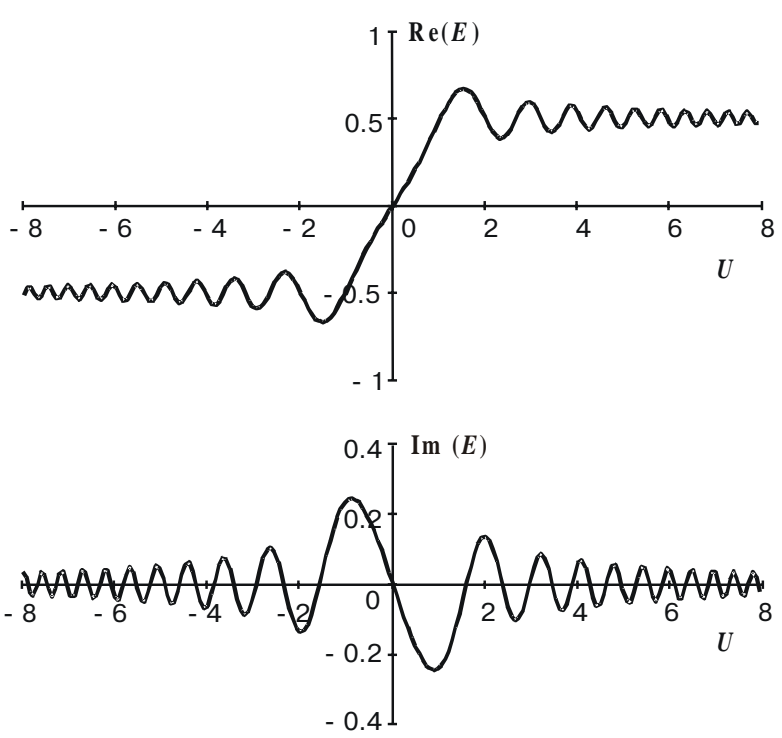

Fig. 5. Real (a) and imaginary (b) part of EDW (projections of Cornu's spiral)

$\operatorname{Re}[E(x, y, z)]=0 \operatorname{Im}[E(x, y, z)]=0$.

Both values zero simultaneously exactly on the edge of the geometrical shadow (Fig. 5), thereby the area of existence of unique in this case field dislocation is the half-plane $U_{o}=0$, coinciding with the said edge. Twodimensional structure of the edge dislocation in such case is a direct consequence of two-dimensional character of this task.

Similar field structure in free state may be formed by using two-slot slit Young experiment [3] at introduction phase shift by $\mathrm{p}$ between both interfering components. In such case, the edge dislocation occurs as a central minimum of the formed pattern. As against to the hidden from immediate observation singularity of the diffraction field, this structure exists self-maintained, that makes it convenient for analysis by the model object.

The main property of the majority of singular structures is their topological resistance to small amplitudephase distortions [6]. So, most inherent to the actual light beams screw dislocations of the wavefront, existing in space in the form of one-dimensional structures - zero amplitude lines - are only deformed or undergo spatial shift rather than disappear under the said distortion.

In contrast to this, two-dimensional dislocations shaped by using half-plane or a pair of slotslit, on the contrary, are extremely sensitive to amplitude-phase distortion generating their field. Really, any however small amplitude or phase deviation of the interfering components in the said Young experiment from equilibrium value leads to inevitable separation of the resulting minimum field on the system axis from the zero level, that, by definition, deprives this field of singularity. As we will see below, such high criticality to wave distortion is characteristic for EDW, too.

\subsection{Problem of experimental EDW isolation}

Experimental EDW isolation from the diffraction field is based on the interference suppression of plane wave component of such field by using wave antiphase thereto $[1,2]$. However, isolation of pure EDW in such case becomes problematic in such case because of a necessity to secure absolutely precise value of amplitude and phase of the suppressing wave. Let us consider this in more detail.

At superposition of antiphase plane wave onto diffraction field availability of edge dislocation in the general field is determined by the following condition:

$\left\{\begin{array}{l}\operatorname{Re}\left[E_{z}^{(E D W)}(U)\right]+\frac{A_{0}}{2}+G \times \cos [\Phi]=0 \\ \operatorname{Im}\left[E_{z}^{(E D W)}(U)\right]+G \times \sin [\Phi]=0\end{array}\right.$,

where $G \times \exp [i \Phi]$-- is the suppressing plane wave field. From the conditions (6)-(7) it follows that isolation of pure EDW requires absolute equality of the suppressing wave amplitude and half amplitude of the initial wave, and these waves being strictly antiphase to each other:

$G=A_{0} / 2, \Phi=\pi$

In actual conditions this requirement may be met only with certain final accuracy, determined by the specific technical possibility of experimental scheme. This means that the isolated field, apart from EDW, almost always contains certain small uncontrollable wave component $g$ $=\mathrm{a} \cdot \exp$ (iф), depriving it of singularity. Considering that component, singularity condition (6) in general form shall be written as follows

$\left\{\begin{array}{l}\operatorname{Re}\left[E_{z}^{(E D W)}(U)+g\right]=0, \\ \operatorname{Im}\left[E_{z}^{(E D W)}(U)+g\right]=0\end{array}\right.$.

In case this condition is met, edge dislocation at the wavefront persists, but its position does not coincide with the edge of the geometrical shadow. In reality, however, it is normally impossible to meet this requirement in practice owing to the above.

More typical situation is shown in Fig. 6, which presents amplitude-phase pattern of the field observed at certain excess amplitude of plane wave $a$, when $\Phi=0$. As it is seen, the main minimum field in this case does not reach zero and is slightly biased from the edge border of the geometrical shadow; at the same time, half-wave leap phase is replaced with its fast, but analytically rounded changes in these limits. However, it is frequently difficult to distinguish pattern visually observed in such case from ideal dislocation.

Let us remember, that apart from immediate isolation of EDW from the diffracted wave it may be obtained otherwise [1,2]. A similar wave, in particular, may be formed directly from the plane with graduated phase shift by $p$ of one of its halves relative to the other or by passing such wave through diffraction lattice, composed of two parts, 


\section{S. Anokhov et al.: Singular peculiarities of a planey wave diffracted ...}
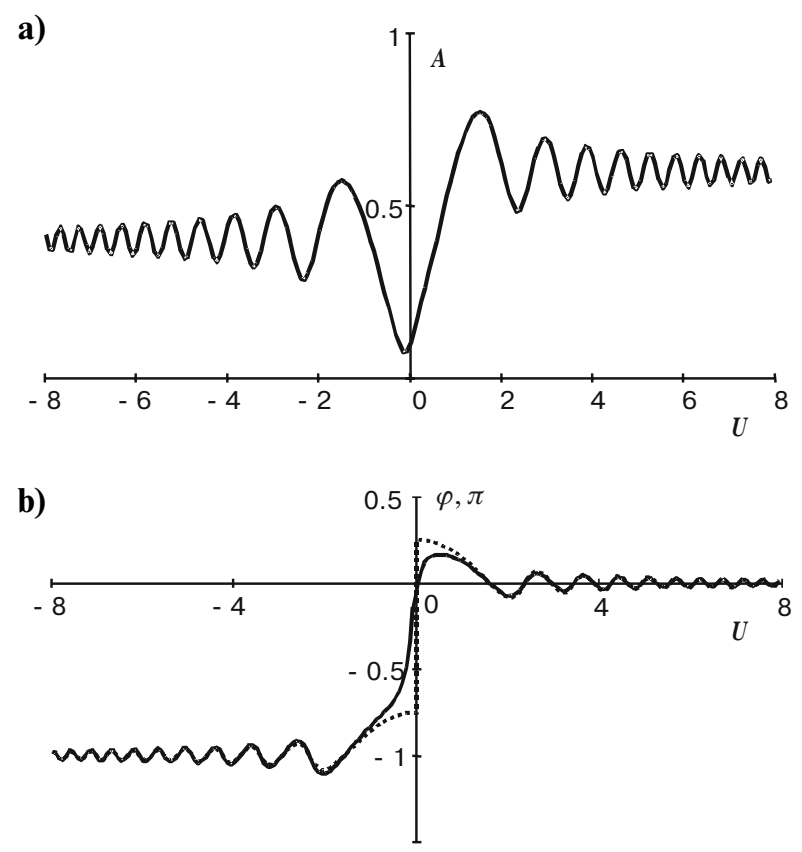

Fig. 6. Transformation of the amplitude (a) and phase (b) of EDW near the geometrical shadow $(a=0.1)$.

shifted one to another by half spatial phase. However, the complete isolation of EDW from the interference remains problematic in this case, too, transformed in final accuracy of the used optical devices manufacturing.

Let us note in conclusion, that the errors of the considered type may be almost completely avoided at isolation of EDW by optical filtration of the diffracted wave. This procedure, however, leads to automatic elimination of natural low-frequency EDW component whose spatial distribution gains in such case «truncated» character (Fig. 3). This circumstance makes such mode wave more similar to actual real light beams while it keeps the main elements of the initial structure of EDW.

\subsection{Reorganization tuning of spatial position of edge dislocation}

As it follows from the equations (7), the coordinate of the isolated edge dislocation may be controlled by changing the suppressing wave parameters. In particular, for such dislocation to be located in an arbitrary point $U_{1}$ parameter of this wave shall be determined by these formulae:

$G=\frac{A_{0}}{2} \times \sqrt{2 \times\left(C^{2}\left(U_{1}\right)+C\left(U_{1}\right)+S^{2}\left(U_{1}\right)+S\left(U_{1}\right)\right)+1}=$ $=\frac{A_{0}}{2} \times \sqrt{J\left(U_{1}\right)}$,

$\Phi=-\operatorname{Arctg}\left[\frac{C\left(U_{1}\right)-S\left(U_{1}\right)}{C\left(U_{1}\right)+S\left(U_{1}\right)+1}\right]=\pi+\Psi\left(U_{1}\right)$ where $J\left(U_{1}\right)$ and $\Psi\left(U_{1}\right)$, are, accordingly, intensity and phase of the diffracted field for the initial plane wave having unit amplitude. The dependencies of $G$ and $\Phi$ from the position of dislocation $U_{l}$ are presented in Fig. 7. Both curves naturally follow well knownwell-known distribution of amplitude and phase (shifted by $p$ ) of the integral diffraction field $[1,2]$ whereby a possibility of interference suppression for this field is provided. Availability of dislocation in point $U_{1}$ may be revealed by direct checking. Having presented therefor the resulting field considering (1) and (10) in this form

$$
\begin{aligned}
& E_{z}(U)=A_{0} \times\left\lfloor\sqrt{J(U)} \exp [\Psi(U)]-\sqrt{J\left(U_{1}\right)} \exp \left[\Psi\left(U_{1}\right)\right]\right\rfloor \\
& \times \exp \left[-i k r \cos \left(\theta-\alpha_{o}\right)\right],
\end{aligned}
$$

we see that simultaneous zeroing of both real and imaginary components of complex amplitude aggregate total field occurs actually in point $U=U_{l}$.

This way, controlling the amplitude and phase of the suppressing wave, the position of the isolated edge dislocation may be changed arbitrarily. In each case for its specific localization, it is required to join EDW with a particular plane wave. By introducing of two such antiphase waves simultaneously into diffraction field (that, obviously, does not change the system) and joining their pairwise with our components, we obtain new combination of plane and singular waves with displaced edge dislocation in the latter. Obviously, the representation of
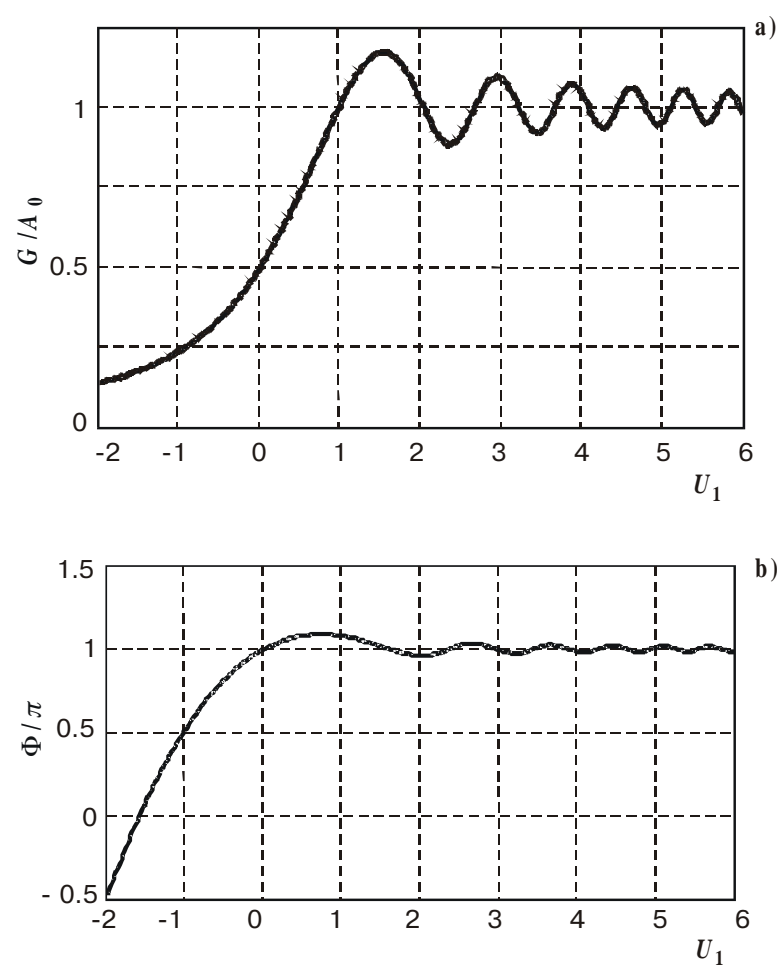

Fig. 7. Amplitude (a) and phase (b) of additional wave depending on the spatial position of edge dislocation. 


\section{S. Anokhov et al.: Singular peculiarities of a planey wave diffracted ...}

diffraction field by such combinations supposes unlimited number of alternatives, deprived, however, of major advantages of using symmetric EDW. Let us note that broad set of such waves may be obtained simultaneously by proceeding to superposition of the fields diffracted on crossed half-planes (see the next section).

In general, dislocation of the considered type is a cylindrical surface with parabola-like generatrix generator (4). Because of asymmetry of general field distribution regarding the very dislocation, this latter, distancing from the screen is displaced along parabola, as if «repulsiverepelling» the edge border of the geometrical shadow. The cylindrical surface degenerates into plane and EDW itself is released in the pure form only in a case when the suppressing wave happens to be twice as less the initial plane wave by amplitude and opposite to phase thereto.

Theoretically estimated field patterns with dislocation position in points $U_{1}= \pm 1$, are presented in Fig. 8a,c, and Fig. 8b compares the distribution of non-distorted EDW. As it is seen, the dislocation bias from the edge of the shadow is accompanied with noticeable lowering of the EDW amplitude involved in this wing with simultaneous uprise of the other wing. At the same time, the nearest extrema of the involved wing undergo certain bias in the same direction as the dislocation while the positions of the extrema of the other wing remains constant.
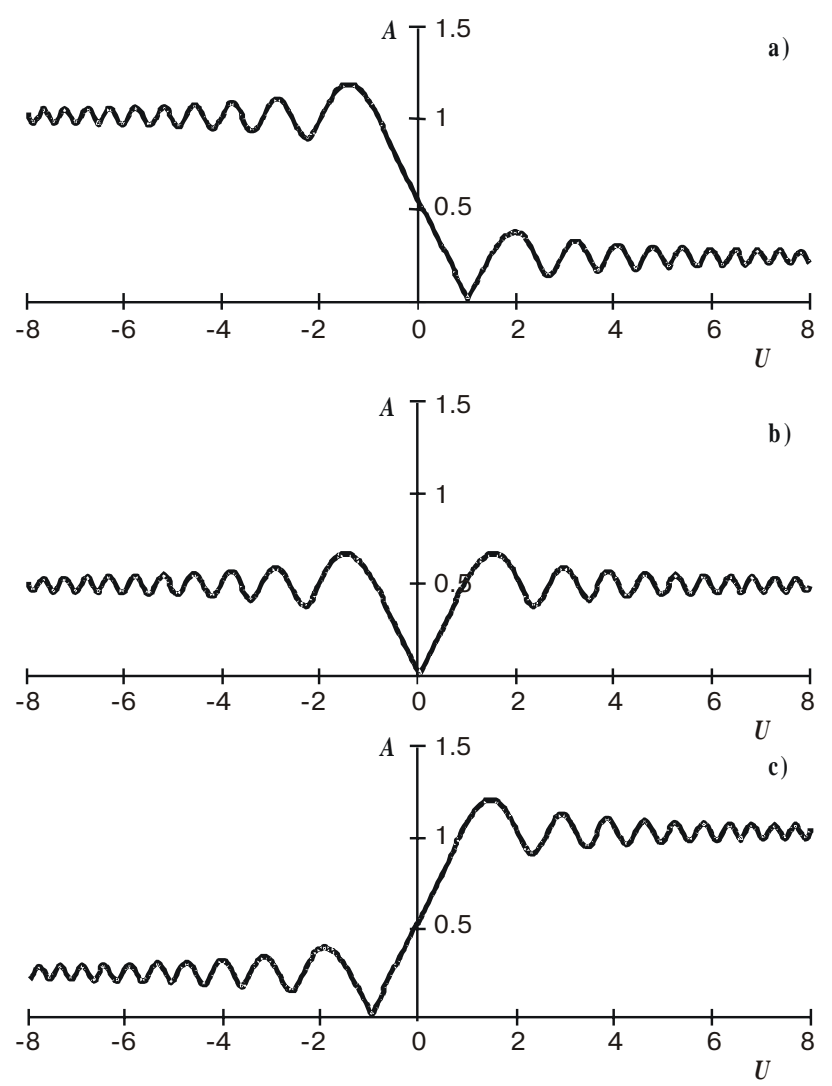

Fig. 8. Evolution of the amplitude distribution of EDW depending on the position of dislocation $U_{1}=1$ (a), $U_{1}=0$ (b), $U_{1}=-1$ (c).

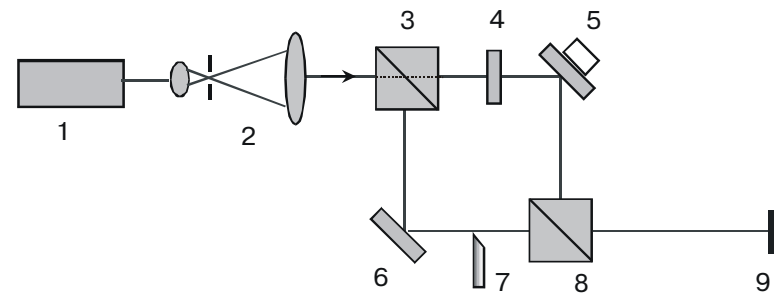

Fig. 9. Experimental set-up: 1 - He-Ne laser, 2 - telescopic beam expander with pinhole (plane-wave former), 3 - beam splitter $(50 \%), 4$ - variable density filter, 5 - mirror mounted on piezodriver, $6-100 \%$ reflecting mirror, 7 - sharp-edge slit, 8 beam splitter $(50 \%), 9$ - image plane with a CCD camera.

Possibility of experimental controlling the position of edge dislocation was explored by using the scheme based on Mach-Zehnder interferometer (Fig. 9). Position of dislocation in the area of light or shadow in such case is reached at suppressing wave amplitude, accordingly, greater or smaller to half amplitude of the initial wave. Typical field patterns at two almost symmetrical positions of edge dislocation with participation of the reference plane wave detecting the phase structure of the field, are presented in Fig. 10.

Let us note that visual identification of half-wave phase leap field at passing through dislocation is complicated here with distinction in the shape of the adjoining areas wavefront - quasiplane, on the side of the attenuated wing front and cylindrical on the side of the strong wing.

\subsection{Superposition of waves diffracted on crossed half-planes}

Having designated $W$ a dimensionless coordinate describing bias dislocation from the edge of geometrical shadow, and introduced two-dimensional function, equal to the difference between two Fresnel's integrals

$$
E(U, W)=\frac{1-i}{\sqrt{2 \pi}}[f(U)-f(W)]=\frac{1-i}{\sqrt{2 \pi}} \int_{W}^{U} \exp \left(i \mu^{2}\right) d \mu
$$

we obtain a wave surface $E(U, W)$ identically equal to the difference between two EDWs, characterized by independent parameters $U$ and $W$ (Fig. 11a). Sections of this surface at $W=$ const are profiles of already familiar singular waves from the family of deformed EDWs with displaced dislocation dealt with in the previous section. The dashed line in this figure shows continuous trajectory of edge dislocation in space $(U, W)$, implementing all possible alternatives of its position.

Experimental simulation of such field may be implemented by interference of two waves, diffracted by half- 


\section{S. Anokhov et al.: Singular peculiarities of a planey wave diffracted ...}

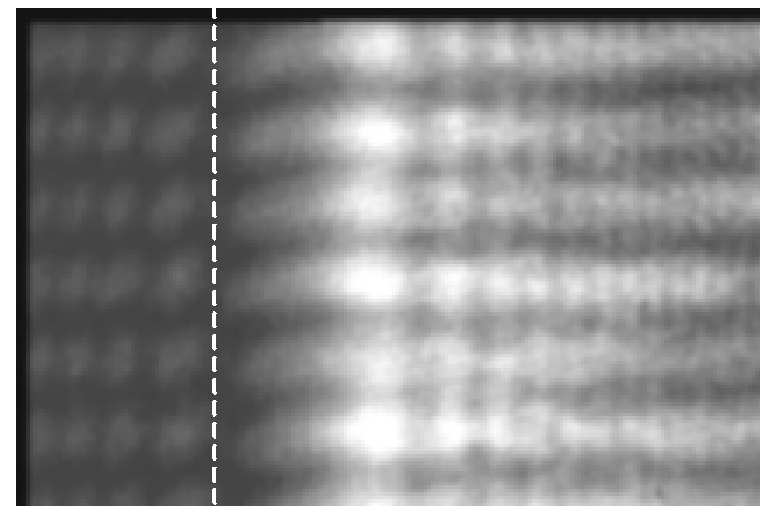

a)

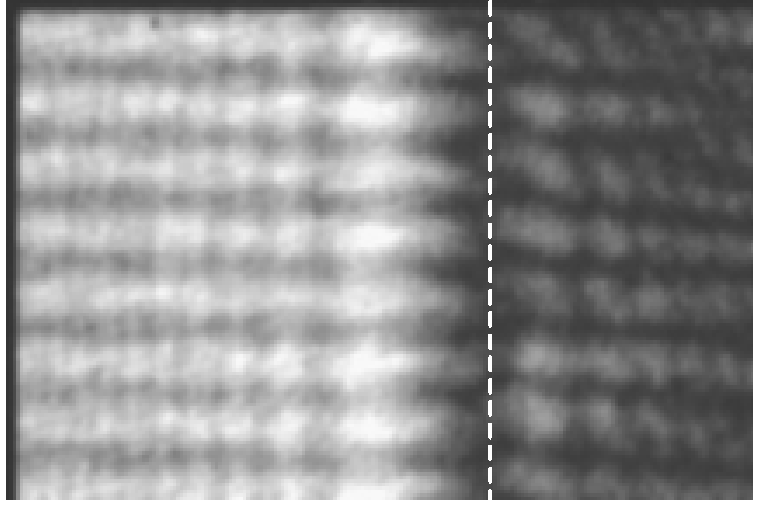

b)

Fig. 10. Experimental (in grey scale) pictures of intensity distribution of interference of the edge dislocation waves with $U_{1}=-1$ (a) and $U_{1}=0.8$ (b) with reference tilted plane wave. The dotted lines pointed out the position of edge dislocation.

planes with orthogonal edges, when introducing the phase shift by $\pi$ between these waves:

$$
\begin{aligned}
& E_{\exp }\left(V_{z}, V_{x}\right)=\left(\frac{A_{o}}{2}+E_{z}^{(E D W)}\right)- \\
& -\left(\frac{A_{o}}{2}+E_{x}^{(E D W)}\right)=E_{z}^{(E D W)}-E_{x}^{(E D W)} .
\end{aligned}
$$

With this purpose in the mind, we supplemented the scheme presented in Fig. 9 with one more screen, edge of which was oriented perpendicularly to that of screen 7 , and located near to piezo-controlled mirror 5. The pat- terns of the field observed in such case are presented in Fig. $11 \mathrm{~b}, \mathrm{c}$ (in the latter case with reference wave). As it is seen, the general field dislocation is oriented as in Fig. 11a, along the diagonal between the edges of halfplane screens. It may be demonstrated that structures of such type are an integral element of diffraction field formed by any rectangular aperture.

Let us note one important circumstance. In this task, polarization of the initial wave in relation to both halfplanes is deliberately unequal that, generally, may not be neglected. In reality, however, Sommerfeld solution for the field behind the half-plane in case of $E$ - and $H$ - polarization of the initial wave differs only with the sign
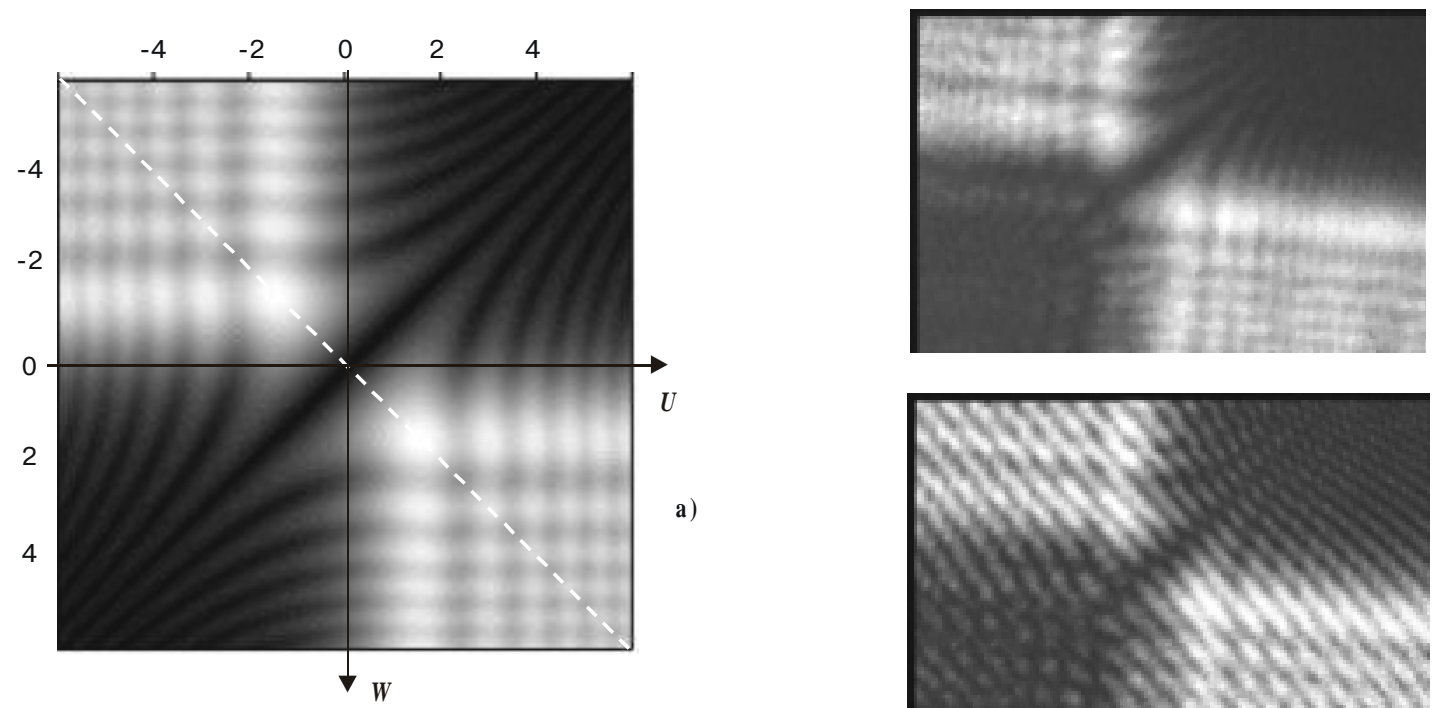

b)

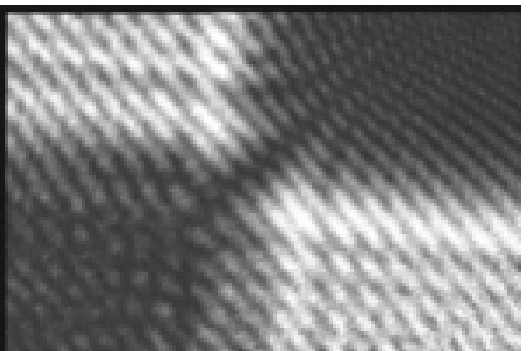

c)

Fig. 11. Theoretical (a) and experimental (b) (in grey scale) pictures of intensity distribution of superposition of two EDW's formed by half-plane with perpendicular edges and the result of interference with reference tilted plane wave (c). Dotted line shows the position of edge dislocation. 


\section{S. Anokhov et al.: Singular peculiarities of a planey wave diffracted ...}

of «evanescentdepressed» field, related with wave, reflected by half-plane [3]. As it is known, amplitude of such field at distancing from the half-plane drops fast, lowering approximately twenty times at a distance of one hundred wave lengths [1,2]. As the used in the experiment screen (as a rule, razor's edge) by its properties far from ideal Sommerfeld half-plane, reflected components of diffraction field are essentially attenuated normally and cannot be recorded. Owing to the above, influence of «evanescentdepressed» field on the EDW so formed may be neglected.

\subsection{Dealing with more complicated apertures}

Criticality of EDW to small wave distortions allows also explaining also specificity of diffraction field evolution at more complicated form of aperture edge. Really, any contortion of rectilinear edge or using more complicated apertures (slotslit, round aperture etc.), inevitably entails spatial overlapping of diffracted singular components from conjugate points of aperture and, automatically, loss of this singularity by them. Joining the said components makes essentially impossible their individual experimental isolation, that, however, hinders no solving of the inverse problem - using such waves for theoretical or experimental synthesis of the diffracted field.

In all considered situations the amplitude of «joined» EDW directly near to the edge of aperture remains close to zero. This wave itself because of the said cross superposition of its singular component constituents is not singular in general, only imitating availability of edge dislocation with deep amplitude fall separating central maximum field thereof. It is the said structure that relates to effect of double shape screen outlining, clearly observed in the area of short-range field at interference suppression of «non-diffracted» components (initial wave of half amplitude) [7] or elimination of low-frequency angular diffraction of field component by other methods $[8,9]$. With distancing from the screen, the contrast of the observed contour worsens fast.

At the same time, the field of «joined» EDW maintains also residual elements of singularity whose spatial position corresponds to constructive interference of all singular components present. Instead of continuous twodimensional surface, representing edge dislocation in case of half-plane, isolated field in this case contains a set of isolated one-dimensional linear dislocation described by condition

$$
\operatorname{Im}\left[\sum_{i} E_{i}^{(E D W)}(U)\right]=0, \operatorname{Re}\left[\sum_{i} E_{i}^{(E D W)}(U)\right]=0,
$$

where $E_{i}^{(E D W)}(U)$ are the singular components formed at diffraction of initial wave on separate quasi-linear areas, component of aperture edge.

Each dislocation is a zero amplitude line bypassing which the field phase shifts by $\pi$. In case of strictly perpendicular orientation of such dislocation to the direc- tion of wave vector, the field structure in the adjoining area may be spot, as pure «edge» dislocation curl. With deviation from cross orientation, this structure all the more transforms into «longitudinal» curl vortex, i.e. screw dislocation [10].

Specific orientation of the shaped dislocation in space depends upon shape of the aperture generating them. Among other apertures special position here is occupied by two most spread types: round aperture and slot slit (or, in more general case, any aperture with rectilinear parallel edge). Formed by them dislocation families is a set of «cross» whose curlsedge dislocation, shape, and axis orientation accurately follow shape and orientation edges itself aperture. Using apertures of other shape (for example, oval or more complicated aperture) distribution of dislocation ceases to correlate with aperture shape being transformed into composite three-dimensional curve.

Spatial location of the whole family of such dislocations normally does not go away for outside the area of the Fresnel diffraction. At the same time, the exclusiveness of round and slit-like apertures is exhibited here, too, in such case condition (14) both for «joined» EDW and for complete diffraction field takes place also in the focal point of the lens, i.e. in the long-range area of the aperture. In particular, dislocation integral field (also concerning to edge [11]) is zero well known distribution, described, accordingly, by the Airy and $\sin (x) / x$ functions.

\section{Conclusions}

The presented results allow to make several generalizations, represented here as run-down.

1. Novelty of our approach to diffraction, in essence, consists of reducing the strict rigorous Sommerfeld solution to two simple concepts: Cornu's spiral and plane wave. Physical embodiment of Cornu's spiral in such case is a singular wave with edge dislocation coinciding with the edge of the geometrical shadow that we called «EDW» (edge dislocation wave).

2. Owing to self-similar character of EDW, its structure in any spatial area remains scalely invariant that considerably simplifies the analysis of diffraction process and, thanks to physical independence and deep qualitative difference of EDW from accompanying it in the diffracted field of the plane wave, are eligible to simple experimental isolation.

3. Owing to high two-dimensional half-plane-shaped dislocation sensitivity to amplitude-phase distortion, its isolation in the pure form, i.e. in absolute isolation from any noise, normally is not achieved in practice. This circumstance, as well as unlimited character, makes EDW similar with plane wave - a fundamental concept of wave optics whose strict practical implementation is essentially impossible.

4. As against traditional physical diffraction model using hypothetical for optics the Huygens-Fresnel secondary waves of Huygens-Fresnel or having discontinu- 


\section{S. Anokhov et al.: Singular peculiarities of a planey wave diffracted ...}

ous Young waves new interpretation of strict rigorous solution is based on quite real wave. Specifically, this circumstance has made possible at last the detailed theoretical and experimental simulation of real diffraction process, and also correct synthesis of field formed by various apertures.

\section{References}

1. A.I. Khizhnyak, S.P. Anokhov, R.A. Lymarenko, M.S. Soskin M.V. Vasnetsov, Proc. SPIE, 3904, pp. 18-25 (1999).

2. A.I. Khizhnyak, S.P. Anokhov, R.A. Lymarenko, M.S. Soskin, M.V. Vasnetsov, JOSA A, 17 (12), p. $2199-2207$ (2000).
3. M. Born, E. Wolf, Principles of optics, 6-th edition, Pergamon Press «Oxford», 1991.

4. S.P. Anokhov, A.I. Khizhnyak, R.A. Lymarenko, // Sem. Phys. Quant. Elect. \& Opt., 3 (1), p. 94-101 (2000).

5. S.P. Anokhov, R.A. Lymarenko, A.I. Khizhnyak, // Ukrain. phys. journ., 46 (1), p. 62-64 (2001).

6. J.F. Nye, M.V. Berry // Proc. R. Soc. Lond. A 336, p. 165190 (1974).

7. S.P. Anokhov, A.I. Khizhnyak, R.A. Lymarenko, M.S. Soskin, // Ukrain. phys. journ., 46 (2), p. 158-160 (2001).

8. P.V. Polyanskii, G.V. Polyanskaya, // J. Opt. Technol., 64 (4), p. 52-63 (1997).

9. P.V. Polyanskii, //Opt. And Spectr., 85 (5), p. $854-858$ (1998)

10. R.A. Lymarenko, M.S. Soskin, A.I. Khizhnyak, S.P. Anokhov, // Proc. SPIE (2000)

11. J.F. Nye, Natural focusing and fine structure of light, Bristol, Philadelphia, 1999. 